\title{
Variability in bone-seeking tracers and imaging protocols for the diagnosis of cardiac amyloidosis: The more the merrier?
}

\author{
Michal Weiler-Sagie, MD, PhD, ${ }^{\mathrm{a}}$ and Simona Ben-Haim, MD, DSc ${ }^{\mathrm{b}, \mathrm{c}}$ \\ a Department of Nuclear Medicine, Rambam Health Care Campus, Haifa, Israel \\ b Department of Medical Biophysics and Nuclear Medicine, Hadassah University Hospital and \\ Hebrew University, Jerusalem, Israel \\ c Institute of Nuclear Medicine, University College London and UCL Hospitals NHS Trust, \\ London, UK
}

Received Jul 28, 2020; accepted Jul 28, 2020

doi: $10.1007 / \mathrm{s} 12350-020-02330-x$

\section{See related article, pp. 307-318}

Cardiac amyloidosis (CA) is a hot topic in nuclear cardiology and molecular imaging has become an important tool in the management of CA due to the unique capability to diagnose transthyretin CA (ATTR) noninvasively. The diagnosis of ATTR that was based in the past on invasive myocardial biopsy can be obtained with high sensitivity (95-100\%) and near perfect specificity (97-99\%), provided plasma cell disease is excluded, ${ }^{1,2}$ by myocardial scintigraphy with three bone-seeking tracers, ${ }^{99 m}$ Tc-3,3-diphosphono-1,2propanodicarboxylic acid (DPD), ${ }^{99 \mathrm{~m}}$ Tc-Pyrophosphate (PYP) and ${ }^{99 \mathrm{~m}}$ Tc-Hydroxymethylene diphosphonate (HMDP). The three tracers perform equally well in the diagnosis of ATTR but not light chain CA (AL), ${ }^{1}$ likely due to calcium binding. ${ }^{3}$ Recently, three new drugs were approved by the US Food and Drug Administration for the treatment of ATTR with clinical studies underway that will likely expand the use of molecular imaging in the diagnosis and management of CA.

In this issue of the Journal of Nuclear Cardiology, Santarelli et al. have retrospectively assessed HMDP studies of 38 patients who underwent a multi-step imaging protocol, designed to detect the earliest time

Reprint requests: Simona Ben-Haim, MD, DSc, Department of Medical Biophysics and Nuclear Medicine, Hadassah University Hospital and Hebrew University, Ein Kerem, Jerusalem 9112001, Israel; simonabh@hadassah.org.il

J Nucl Cardiol 2022;29:319-22.

$1071-3581 / \$ 34.00$

Copyright (c) 2020 American Society of Nuclear Cardiology. point to diagnose ATTR with high sensitivity and specificity. ${ }^{4}$ Dynamic imaging for as little as $4.5 \mathrm{~min}$, as well as static images as early as 3.5 min after injection, both performed as well as late imaging, 1 to $3 \mathrm{~h}$ after injection. These early images were not subjected to visual interpretation. Rather, regions of interest (ROI) were drawn around structures representing uptake in the blood pool, the heart, skeleton and background. Timeactivity curves (TAC) of heart uptake divided by bone uptake $\left(\mathrm{TAC}_{\text {Heart/Bone }}\right)$ for time $>6 \mathrm{~min}$ and a retention index derived from these data $\left(\mathrm{RI}_{\text {heart-bone }}\right)$ for time $>10$ min distinguished between patients with ATTR and controls with a good intra- and inter-observer consistency. ${ }^{4}$

The results of this study suggest that early planar HMDP scintigraphy, either static or dynamic, may replace late imaging in patients with suspected cardiac amyloidosis. This would be good news to patients, who would benefit from the shortened time in the clinic. However, the altered protocol will require additional validation. The number of patients in present study is small and the study population is selective. None of the patients included in the study had evidence of a monoclonal gammopathy and patients with ischemic heart disease, chronic liver disease or severe renal failure were excluded. Most of the assessed for suspected ATTR are elderly, as is the case in the current report (age $82 \pm 6$ years). These co-morbidities are common at an older age, and typical also for patients with suspected ATTR.

Another unique feature of the study population is that there were no patients with grade 1 uptake in the myocardium, defined as uptake less than bone uptake. Studies with grade 1 uptake are reported as equivocal. ${ }^{5}$ On planar images, it may be difficult to determine if tracer uptake is localized in the myocardium or in the 
blood pool. In such cases, single photon emission tomography (SPECT) has an important role. This may become even more challenging in early imaging, a topic that should be addressed in future studies. Galat et al. ${ }^{6}$ were the first to report the ability of early HMDP planar images to accurately predict late findings. They reported, 9 of 135 (7\%) patients had grade 1 myocardial uptake, one of them was correctly identified as suffering from ATTR by visual scoring of the early image but not by a heart-to-mediastinum ratio of the early image.

\section{BONE-SEEKING TRACERS-THE MORE THE MERRIER?}

The search for better tracers and improved imaging protocols is driven by the motivation to improve study performance. However, with the excellent diagnostic performance for differentiating ATTR from light chain cardiac amyloidosis (AL), $100 \%$ specificity for diagnosis of ATTR in patients with grade $2 / 3$ cardiac uptake of all three bone-seeking tracers (DPD, PYP and HMDP) and no evidence of monoclonal gammopathy, ${ }^{1}$ there seems to be little room for improvement. Although the number of patients scanned with each tracer was not even (DPD 877, PYP 199 and HMDP 141) further studies have established the use of all 3 tracers, ${ }^{2}$ also recommended in current multi-disciplinary consensus recommendations for imaging of cardiac amyloidosis. ${ }^{5}$

All three tracers perform very well in the management of CA-differentiating between ATTR and AL. The choice of tracer is mostly a result of regional availability. Whereas in the USA only PYP is approved by the FDA, in other countries DPD, HMDP and HDP are available. In countries where more than one tracer is available, the cost, ease of interpretation and protocol differences may play a role in determining which tracer to use.

Is one tracer better than others? There are no studies directly comparing these three tracers in patients with suspected CA. In addition, the mechanism by which bone-seeking tracers bind to TTR fibrils and not to light chain fibrils is unclear. The hypothesis that there are more microcalcifications in ATTR compared to AL has very little supporting evidence. There is a single study describing a greater density of microcalcifications in endomyocardial biopsies of eight ATTR patients compared to seven AL patients. ${ }^{3}$ To the best of our knowledge, there are no studies comparing the performance of the tracers in animal models of ATTR.

Not all bone-seeking tracers are helpful for ATTR imaging. Although they are all incorporated to the hydroxyapatite in the mineralization process, the exact mechanism of uptake is unclear. The tracers differ in structure, protein binding, clearance from the blood, urinary excretion and in their intrinsic affinity to bone (Table 1, Figure 1). ${ }^{7-10}$ In stark contrast to PYP, DPD and HMDP, the most commonly used tracer for bone imaging, ${ }^{99 \mathrm{~m}} \mathrm{Tc}$ methylene diphosphonate (MDP), shows no uptake in ATTR. ${ }^{11,12}$ Similarly, the PET tracer ${ }^{18} \mathrm{FNa}$, despite superior resolution and sensitivity for bone lesions, has low sensitivity for ATTR. ${ }^{13,14}$ MDP and ${ }^{18} \mathrm{FNa}$ are cleared quickly from the blood and soft tissues, ideally suited for bone imaging. These properties may, however, hinder their ability to interact with the presumed microcalcifications in the TTR fibrils. The degree of availability of the different tracers for myocardial incorporation and their specific affinity to fibril microcalcifications have not been studied.

\section{IMAGING PROTOCOLS-THE MORE THE MERRIER!}

In the multi-center trial including 1217 patients, reported by Gillmore et al., a single imaging protocol was used for all three tracers, late planar imaging, ${ }^{1}$ yielding fabulous results. Other protocols are evolving, adapting to the unique characteristics of each tracer. The protocols differ in technique: dynamic imaging, planar imaging, whole body versus a single field of view (FOV) of the chest, and the use of SPECT or SPECT/CT. The more imaging protocols the more time for the patient under the camera, but also the more information available for the interpreting physician on which to base the diagnosis.

The most important evolution in protocol has been the addition of SPECT to planar imaging. ${ }^{15,16}$ SPECT aids in better localization of tracer activity to the myocardium as opposed to the left ventricular blood pool ${ }^{17}$ and helps avoiding pitfalls. ${ }^{18}$ Imaging the whole body as opposed to a single FOV of the chest allows evaluation of soft tissue uptake, described mainly in DPD and HMDP.

Masri et al. showed comparable diagnostic performance in 233 patients who had planar and SPECT imaging at $1 \mathrm{~h}$ and $3 \mathrm{~h} .{ }^{17}$ For planar images grades $1-2$, SPECT was needed to make the diagnosis. SPECT/CT enables quantitation ${ }^{19,20}$ and localization of uptake in soft tissue. ${ }^{21,22}$

Another variation of the protocol is timing of the imaging, from $10 \mathrm{~min}$ as suggested by Santarelli et al. in this issue, ${ }^{4}$ to $3 \mathrm{~h}$ after the injection, the most validated time point. DPD imaging is most commonly performed at $3 \mathrm{~h}$ after injection (the past practice of early and late imaging has mostly been abandoned), ${ }^{16}$ whereas PYP imaging is recommended at 1-h post-injection with further imaging at $3 \mathrm{~h}$ in cases of high background, i.e. due to renal failure. ${ }^{22}$ 
Table 1. Properties of bone-seeking radiotracers ${ }^{7-10}$

\begin{tabular}{|c|c|c|c|c|c|}
\hline & PYP & DPD & HMDP & MDP & NaF \\
\hline Structure & $\begin{array}{l}\text { Inorganic P-O-P } \\
\text { diphosphate }\end{array}$ & \multicolumn{3}{|c|}{ Organic P-C-P diphosphonate } & Inorganic compound \\
\hline $\begin{array}{l}\text { Mechanism } \\
\text { of bone uptake }\end{array}$ & \multicolumn{4}{|c|}{ Ion exchange and chemisorption to hydroxyapatite } & $\begin{array}{l}\text { Exchange of hydroxyl } \\
\text { groups on } \\
\text { hydroxyapatite to form } \\
\text { fluorapatite }\end{array}$ \\
\hline Protein binding & High & Low & & & \\
\hline \multirow[t]{3}{*}{$\begin{array}{l}\text { Clearance from } \\
\text { blood }\end{array}$} & 2-phase: & 2-phase: & $\begin{array}{l}\text { Similar } \\
\text { to MDP }\end{array}$ & 3-phase: & 2-phase: \\
\hline & $\mathrm{T}_{1 / 2} 13.6 \mathrm{~min}$ & $\mathrm{~T}_{1 / 2} 15 \mathrm{~min}$ & & $\begin{array}{l}\mathrm{T}_{1 / 2} 3.5 \mathrm{~min}, \\
\mathrm{~T}_{1 / 2} 37 \mathrm{~min},\end{array}$ & $\mathrm{~T}_{1 / 2} 24 \mathrm{~min}$ \\
\hline & $\mathrm{T}_{1 / 2} 380 \mathrm{~min}$ & $\mathrm{~T}_{1 / 2} 100 \mathrm{~min}$ & & $\begin{array}{l}\mathrm{CH}_{6} \mathrm{O}_{6} \mathrm{P}_{2} \\
\mathrm{~T}_{1 / 2} 144 \min \end{array}$ & $\mathrm{T}_{1 / 2} 156 \mathrm{~min}$ \\
\hline Molecular formula & $\mathrm{H}_{4} \mathrm{O}_{7} \mathrm{P}_{2}$ & $\mathrm{C}_{5} \mathrm{H}_{10} \mathrm{O}_{10} \mathrm{P}_{2}$ & $\mathrm{CH}_{6} \mathrm{O}_{7} \mathrm{P}_{2}$ & $\mathrm{CH}_{6} \mathrm{O}_{6} \mathrm{P}_{2}$ & $\mathrm{FNa}$ \\
\hline $\begin{array}{l}\text { Topological polar } \\
\text { surface area }\left(\AA^{2}\right)\end{array}$ & 124 & 190 & 135 & 115 & 0 \\
\hline Additional features & $\begin{array}{l}\text { Binds to RBC } \\
\text { Hydrolyzed in } \\
\text { liver }\end{array}$ & $\begin{array}{l}\text { Renal } \\
\text { reabsorption }\end{array}$ & & & Binds to RBC \\
\hline
\end{tabular}

PYP, ${ }^{99 m}$ Tc-pyrophosphate; DPD, ${ }^{99 m} \mathrm{Tc}-3,3$-diphosphono-1,2-propanodicarboxylic acid, HMDP, ${ }^{99 m} \mathrm{Tc}-\mathrm{Hydroxymethylene}$ diphosphonate; MDP, ${ }^{99 \mathrm{~m}} \mathrm{Tc}$ methylene diphosphonate; NaF, ${ }^{18} \mathrm{~F}$-sodium fluoride

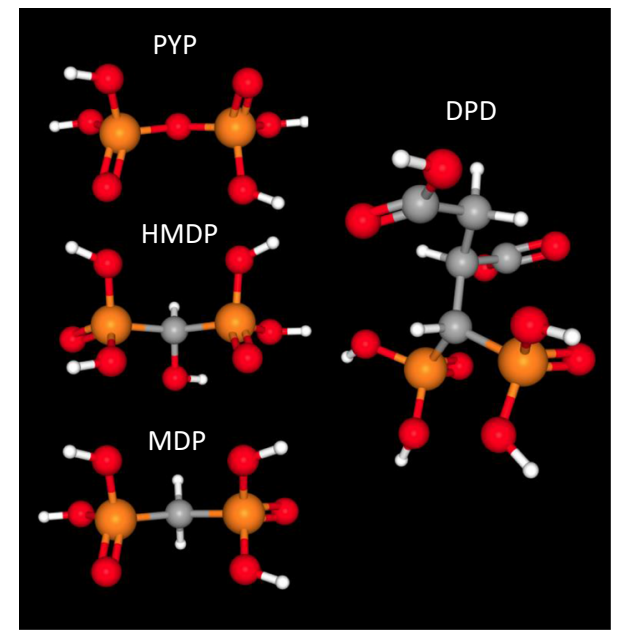

Figure 1. 3D chemical structure models of bone-seeking tracers. ${ }^{3}$ Hydrogen $(\mathrm{H})$ atoms in white. Oxygen $(\mathrm{O})$ atoms in red. Carbon $(\mathrm{C})$ atoms in grey. Phosphor $(\mathrm{P})$ atoms in orange.

As suggested by Santarelli et al. and previously by Galat et al., ${ }^{6}$ HMDP imaging performed as early as at 10 min after injection may enable diagnosis of ATTR. If validated in larger, more varied populations, HMDP may become the most attractive tracer of the three. Shortening the patient visit in the department from a 2 to $3 \mathrm{~h}$ to $10 \mathrm{~min}$ has great impact on patient comfort and departmental workflow. As attractive as this may be, the most important aspect of imaging is its diagnostic confidence, and shortening the time of the study should not compromise the quality of the scan.

With new developments and novel therapeutic agents there is a need for a non-invasive imaging modality to monitor disease activity. Molecular Imaging techniques should be investigated not only in the diagnosis but also in assessment of response to therapy. Time-activity curves from dynamic imaging, as described in present study, as well as quantitative data from SPECT/CT acquisitions may have a role in serial imaging and disease monitoring and further research is warranted.

\section{References}

1. Gillmore JD, Maurer MS, Falk RH, Merlini G, Damy T, Dispenzieri A, Wechalekar AD, Berk JL, Quarta CC, Grogan M, Lachmann HJ. Nonbiopsy diagnosis of cardiac transthyretin amyloidosis. Circulation 2016;133(24):2404-12.

2. Treglia G, Glaudemans AWJM, Bertagna F, et al. Diagnostic accuracy of bone scintigraphy in the assessment of cardiac transthyretin-related amyloidosis: a bivariate meta-analysis. Eur J Nucl Med Mol Imaging 2018;45:1945-55.

3. Stats MA, Stone JR. Varying levels of small microcalcifications and macrophages in ATTR and AL cardiac amyloidosis: 
implications for utilizing nuclear medicine studies to subtype amyloidosis. Cardiovasc Pathol. 2016;25:413-7.

4. Santarelli et al. (J Nucl Cardiol....)

5. Dorbala S, Ando Y, Bokhari S, et al. ASNC/AHA/ASE/EANM/ HFSA/ISA/SCMR/SNMMI Expert Consensus Recommendations for Multimodality Imaging in Cardiac Amyloidosis: Part 1 of 2Evidence Base and Standardized Methods of Imaging. Journal of Nuclear Cardiology

6. Galat A, Van der Gucht A, Guellich A, Bodez D, Cottereau AS, Guendouz S, Hittinger L, Dubois-Randé JL, Plante-Bordeneuve V, Itti E, Meignan M. Early phase 99Tc-HMDP scintigraphy for the diagnosis and typing of cardiac amyloidosis. JACC 2017;10(5):601-3.

7. Fogelman I. Skeletal uptake of diphosphonate: A review. Eur J Nucl Med 1980;5(6):473-6.

8. Fogelman I. Diphosphonate bone scanning agents-Current concepts. Eur J Nucl Med 1982;7(6):506-9.

9. Subramanian G, McAfee JG, Blair RJ, Kallfelz FA, Thomas FD. Technetium-99m-methylene diphosphonate-A superior agent for skeletal imaging: Comparison with other technetium complexes. J Nucl Med 1975;16:744-55.

10. https://pubchem.ncbi.nlm.nih.gov/ compounds 1023, 189891, 27304, 16124, 23690531. Accessed July 17, 2020.

11. Perugini E, Guidalotti PL, Salvi F, et al. Noninvasive etiologic diagnosis of cardiac amyloidosis using 99mTc-3,3-diphosphono1,2-Propanodicarboxylic Acid scintigraphy. J Am Coll Cardiol 2005;46:1076-84.

12. Cuscaden C, Ramsay SC, Prasad S, Goodwin B, Smith J. Estimation of prevalence of transthyretin (ATTR) cardiac amyloidosis in an Australian subpopulation using bone scans with echocardiography and clinical correlation. J. Nucl. Cardiol. 2020. https://d oi.org/10.1007/s12350-020-02152-x.

13. Martineau P, Finnerty V, Giraldeau G, Authier S, Harel F, Pelletier-Galarneau M. Examining the sensitivity of $18 \mathrm{~F}-\mathrm{NaF}$ PET for the imaging of cardiac amyloidosis. J. Nucl. Cardiol. 2019. http s://doi.org/10.1007/s12350-019-01675-2.
14. Ng QKT, Sethi P, Saunders TA, Pampaloni MH, Flavell RR. Discordant findings on ${ }^{18} \mathrm{~F}-\mathrm{NaF}$ and ${ }^{99 \mathrm{~m}} \mathrm{Tc}-\mathrm{HDP}$ bone scans in a patient with ATTR cardiac amyloidosis. Clin Nucl Med 2018;43:e89-92.

15. https://www.asnc.org/Files/Practice\%20Resources/Practice\%20Po ints/ASNC\%20Practice\%20Point-99mTechnetiumPyrophosphateI maging2019.pdf

16. https://www.asnc.org/files/19110\%20ASNC\%20AND\%20EAN M\%20Amyloidosis\%20Practice\%20Points\%20WEB(2).pdf

17. Masri A, Bukhari S, Ahmad S, et al. Efficient 1-Hour Technetium$99 \mathrm{~m}$ pyrophosphate imaging protocol for the diagnosis of transthyretin cardiac amyloidosis. Circulation 2020;13:e010249.

18. Ochi Y, Kubo T, Nakashima $\mathrm{Y}$, et al. A case report of an uncommon presentation of 99mtechnetium pyrophosphate scintigraphy in transthyretin cardiac amyloidosis: A potential diagnostic pitfall, pseudo-positive or pseudo-negative? J Cardiol Cases 2020;21:50-3.

19. Caobelli F, Braun M, Haaf P, Wild D, Zellweger MJ. Quantitative ${ }^{99 m}$ Tc-DPD SPECT/CT in patients with suspected ATTR cardiac amyloidosis: Feasibility and correlation with visual scores. Cardiol: J. Nucl; 2019.

20. Manrique A, Dudoignon D, Brun S, et al. Quantification of myocardial ${ }^{99 \mathrm{~m}} \mathrm{Tc}$-labeled bisphosphonate uptake with cadmium zinc telluride camera in patients with transthyretin-related cardiac amyloidosis. Eur J Nucl Med Mol Imaging Res. 2019;9:117.

21. Malka N, Abulizi M, Kharoubi M, et al. Extracardiac soft tissue uptake, evidenced on early 99mTc-HMDP SPECT/CT, helps typing cardiac amyloidosis and demonstrates high prognostic value. Eur J Nucl Med Mol Imaging 2020.

22. Bokhari S, Morgenstern R, Weinberg R, et al. Standardization of ${ }^{99 \mathrm{~m}}$ Technetium pyrophosphate imaging methodology to diagnose TTR cardiac amyloidosis. J Nucl Cardiol 2018;25:181-90.

Publisher's Note Springer Nature remains neutral with regard to jurisdictional claims in published maps and institutional affiliations. 